Andrews University

Digital Commons @ Andrews University

Faculty Publications

$12-1-1997$

\title{
The L3 Neuron and an Associated Prothoracic Network are Involved in Calling Song Recognition by Female Crickets
}

\author{
John Stout \\ Andrews University, stout@andrews.edu \\ Nathan Carlson \\ Andrews University \\ Hilary Bingol \\ Andrews University \\ James Ramseier \\ Andrews University \\ Michael Bronsert \\ Andrews University
}

See next page for additional authors

Follow this and additional works at: https://digitalcommons.andrews.edu/pubs

Part of the Entomology Commons

\section{Recommended Citation}

Stout, John; Carlson, Nathan; Bingol, Hilary; Ramseier, James; Bronsert, Michael; and Atkins, Gordon, "The L3 Neuron and an Associated Prothoracic Network are Involved in Calling Song Recognition by Female Crickets" (1997). Faculty Publications. 2535.

https://digitalcommons.andrews.edu/pubs/2535

This Conference Proceeding is brought to you for free and open access by Digital Commons @ Andrews University. It has been accepted for inclusion in Faculty Publications by an authorized administrator of Digital Commons @ Andrews University. For more information, please contact repository@andrews.edu. 


\section{Authors}

John Stout, Nathan Carlson, Hilary Bingol, James Ramseier, Michael Bronsert, and Gordon Atkins 


\title{
The L3 neuron and an associated prothoracic network are involved in calling song recognition by female crickets
}

\section{JOHN STOUT, NATHAN CARLSON, HILARY BINGOL, JAMES RAMSEIER MICHAEL BRONSERT and GORDON ATKINS}

\author{
Biology Department, Andrews University, Berrien Springs, MI 49104, USA
}

\begin{abstract}
In young virgin Acheta domesticus females, the spiking response of the prothoracic L3 auditory interneuron discriminates between calling songs (CSs) with phonotactically attractive and unattractive syllable periods (SPs), which parallels phonotactic discrimination. Presentation of a CS with an originally attractive SP, but with the intensity modulated so as to minimize L3's selective response, results in a CS with little phonotactic attractiveness. Conversely, a CS with an originally unattractive SP becomes much more attractive when the CS is intensity modulated in ways that duplicate L3's selective response. L3's discriminatory response to CS SP deteriorates with age, in parallel with decreased phonotactic selectiveness (females, older than 14 days, typically are unselective for CS SPs). SP-selective processing, which was not apparent in these old L3s, is immediately restored by removing the contralateral ear. SP-specific information is resident in a network of neurons within the prothoracic ganglion that results in the SP selective responses of the L3 neuron in young females. Changes in the SP-selective responses of the L3 neuron are highly correlated with corresponding changes in the female's phonotactically selective behavior.
\end{abstract}

KEY WORDS: L3 neuron; Acheta domesticus; calling song

\section{Introduction}

The phonotactic response of female crickets to models of their conspecific male's calling song and the discrimination of these calls from models of the calls of males of other species has been demonstrated for a number of cricket species (e.g. Thorson et al., 1982, Stout et al., 1983, Doherty 1985, Pollack and Hoy 1981). Syllable period (SP) of the calling song (CS) has been demonstrated to be the most important call parameter used by females in making this discrimination between calls of different males for a number of species (Doherty 1985) including Acheta domesticus (Stout et al., 1983, Stout and McGhee 1988; the calling song of conspecific males has syllable periods ranging between 45 and $70 \mathrm{~ms}$ and a carrier frequency of 5 $\mathrm{kHz}$ ). Thus, the female cricket's recognition of the conspecific male's CS has become an important model system for evaluating the nervous system processing that underlies the encoding for and recognition of signals that communicate information about the sender's behavioral state to conspecifics.

Numerous studies have demonstrated discrimination by female crickets of the conspecific male's call from those of other species (see above). However, the nervous system processing underlying recognition of the conspecific call has been more difficult to evaluate. Schildberger (1984) described neurons in the brain of female Gryllus campestris that responded selectively to the SP of the conspecific male's call. The output of one of these neurons (BNC2a) matched the females behavioral selectiveness and could be responsible for call recognition. However, it has not been possible to evaluate experimentally whether the SP-selective output of the BNC2a neuron is causally related to the female's (G. campestris) selective phonotaxis. Pires and Hoy (1992) demonstrated that for female G. firmus, processing of auditory information, leading to calling song recognition, occurred in both the prothoracic ganglion and the brain. Atkins et al. (1989) described a prothoracic auditory interneuron (termed the L3) in female $A$. domesticus whose selective response to the SP of model CSs also matched the females phonotactic selectiveness.

The purpose of this study is to evaluate whether the correlations between the SP-selective response of the $\mathrm{L} 3$ neuron in female $A$. domesticus might be causally related to the female's selective phonotactic response to the models of the male's calling song. The factors that underlie the selective response of the L3 neuron to the SP of model calls are also considered.

\section{Material and methods}

\section{Animals and care}

Female A. domesticus, purchased as 3 to 4-week-old nymphs from Fluker's Cricket Farm, were raised to 
adults at $22^{\circ} \mathrm{C}$ with a 12:12 LD light cycle (on at 06:00). Cricket chow and water or potatoes were supplied ad libitum. Every day, females that had molted to adults within the previous $24 \mathrm{hr}$ were isolated and maintained under the above conditions but separate from the nymphs. Newly molted adult males were removed each day.

\section{Behavior experiments}

Phonotaxis of female crickets was tested in a circular arena (Walikonis et al., 1991) with a centrally located, omnidirectional loudspeaker. Model CSs $(5 \mathrm{kHz})$ were presented at an intensity of $70 \mathrm{~dB}$ at the edge of the arena for the phonotaxis shown in Fig. 1 and at $90 \mathrm{~dB}$ (100\% syllable amplitude) for the phonotaxis shown in Figs 2 and 3. A phonotactic response was defined as reaching the central loudspeaker within $5 \mathrm{~min}$ of stimulus onset. Synthetic CSs were either produced with a custom-made signal generator or synthesized using a computer (Macintosh II).

\section{Electrophysiology}

Following determination of a female's phonotactic threshold (described above) the threshold (defined as production of a mean of at least one spike in response to each syllable of the CS) of the L3 auditory interneuron was determined in the same individual, using either intracellular or extracellular recording techniques.

Intracellular recordings. Recordings, Lucifer-yellow staining, and morphological identification of the auditory interneurons were performed as described by Atkins et al., (1989).

Extracellular recordings. A small incision was made on the ventral surface of the neck to expose the neck connectives after the cricket was immobilized, ventral side up, on a wax block in a manner similar to that described for intracellular recordings (Atkins et al., 1989). The tip of a suction electrode was placed along side of a desheathed connective and positioned to record from $5 \mathrm{kHz}$-tuned units. Of the three known prothoracic, ascending units in the neck connective, only L3 has a threshold of 65 to $70 \mathrm{~dB}$ at $5 \mathrm{kHz}$ and 55 to $65 \mathrm{~dB}$ at $16 \mathrm{kHz}$ and its unique response properties (Atkins et al., 1989) - thus L1 and L2 (Stout et al., 1985 ) could be easily found and avoided.

Sound stimulation. Model CSs were played through one of a pair of loud speakers (matched pairs of Realistic $\# 40-1379$ piezo high frequency speakers). Sound intensities were calibrated $( \pm 2 \mathrm{~dB})$ using a Heath real time spectrum analyzer (AD-1308). Synthetic CSs were either produced with a custom-made signal generator or synthesized using a computer (Macintosh 7100).

\section{Results}

Adult female A. domesticus ( 3 - 7 days old) most frequently responded with positive phonotaxis to model CSs that reproduce the SP (Fig. 1A, $50 \mathrm{~ms}$ ) that is most characteristic of the male's CS. The range of SPs that were most attractive for the females shown in Fig. $1 \mathrm{~A}$ is very similar to the SP selectiveness already shown for young female A. domesticus (Stout et al, 1983, Walikonis et al., 1991, Stout et al., 1992) and matches the range of SPs produced by males. The L3 neuron responded to a CS with an SP of $50 \mathrm{~ms}$ by decreasing maximally the number of action potentials it produced in response to the second and third syllables of a CS below the number produced in response to the first syllable (Fig. 1A). This decrement in responses is defined as the percent decrease in the number of action potentials produced in response to the third syllable, as compared to the first syllable. L3's response decrement was maximal $(46 \%)$ in response to CSs that were most behaviorally attractive to adult females and minimal $(27 \%)$ to CSs that were least attractive.

Older females ( 21 - 28 days) responded phonotactically at high rates, showing little discrimination between CSs with SPs ranging between 30 and $90 \mathrm{~ms}$ (Fig. 1B). The L3 neuron in females of this age demonstrated little decrement ( 8 to $20 \%$ ) in its responses and thus did not discriminate between CSs with the same range of SPs.

Five-day-old females that were selective for CS SP (data not shown), responded phonotactically to SPs of $50 \mathrm{~ms}$ (Fig. 2A, 100\%). When these females were tested with a series of CSs with an SP of $50 \mathrm{~ms}$, only $25 \%$ of the females that responded to a CS with all syllables produced at the same intensity (100\%) responded to CSs in which the first syllable was produced at $50 \%$ of the amplitude of the other syllables. Rates for positive phonotaxis increased in response to first syllable amplitudes that were greater or smaller that $50 \%$ and were maximal when the first syllable amplitude was $10 \%$ or $100 \%$ (Fig. 2A).

The L3 neuron's decrement was greatest in response to CSs with a first syllable amplitude of $100 \%$ and least when the first syllable amplitude was $50 \%$. Response decrement increased when the first syllable amplitude was greater or smaller than $50 \%$ (Fig 2A, $B)$. The L3 neuron did not respond to the first syllable when its amplitude was $10 \%$ and response decrement was calculated based on the response to the second and third syllables. The females most frequently selected a model CS for phonotactic responses that L3 neurons responded to with maximum decrement (Fig. 2B). Thus, as first syllable amplitude was modulated, 

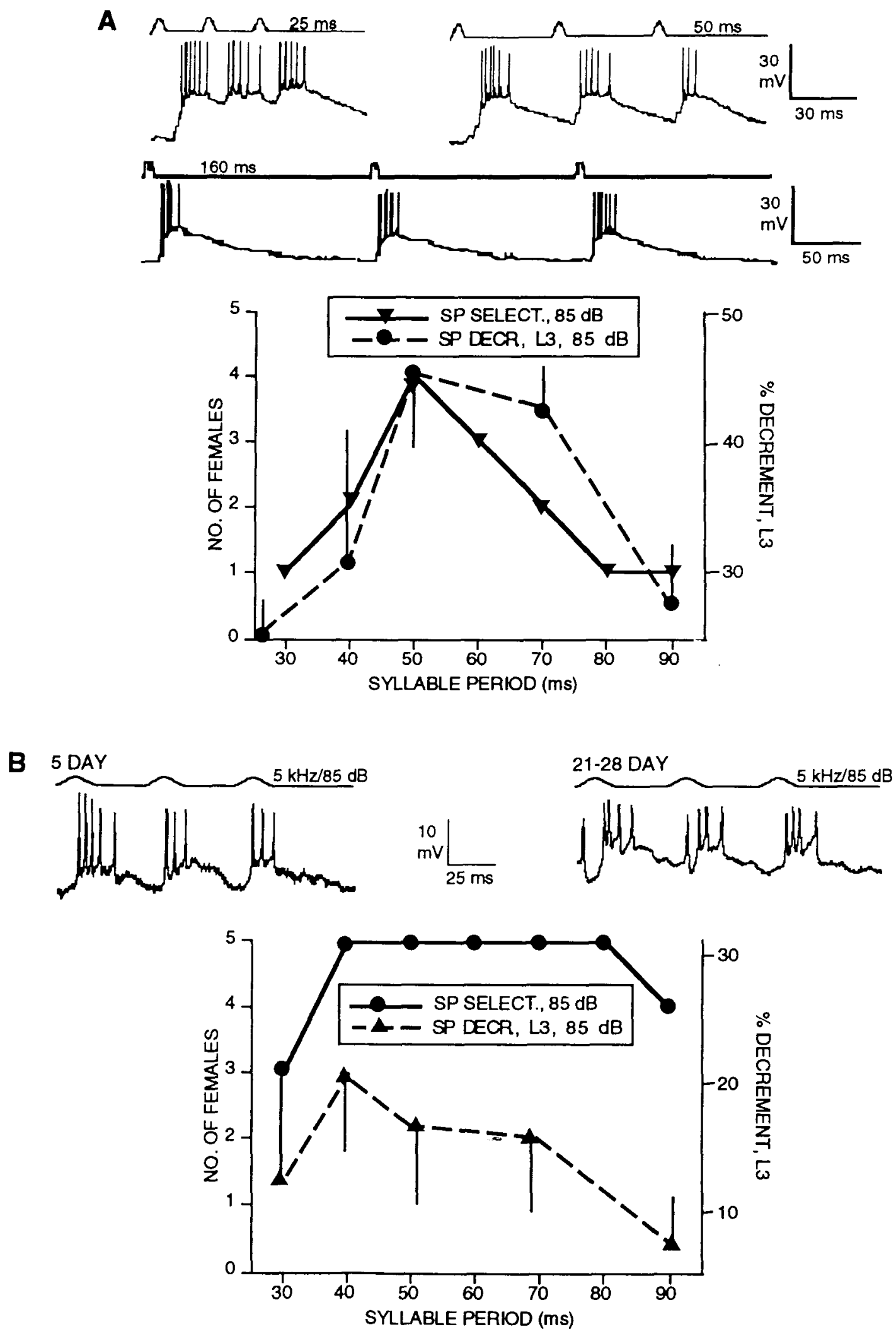

Fig. 1. The correlations between L3's SP-selective responses and 5-day-old female crickets' SP-selective phonotactic responses to model calling songs. A. Intracellular recording of the $\mathrm{L} 3$ neuron's responses to model $\mathrm{CSs}$ with the indicated $S P s$, produced at $5 \mathrm{kHz}, 85 \mathrm{~dB}$. The graph compares the mean SP-selective decrementing responses of $L 3$ neurons (5-day-old females, $n=9)$ and the phonotactic selectiveness of five, 5 day-old females. B. Intracellular recording of the $L 3$ neuron's responses to model CSs (50 ms SP) in young and old females. The graph compares the mean decrementing responses of $L 3$ neurons in 28-day-old females $(n=8)$ and the phonotactic selectiveness of five, 28-day-old females. Bars associated with means indicate the standard errors. 
A
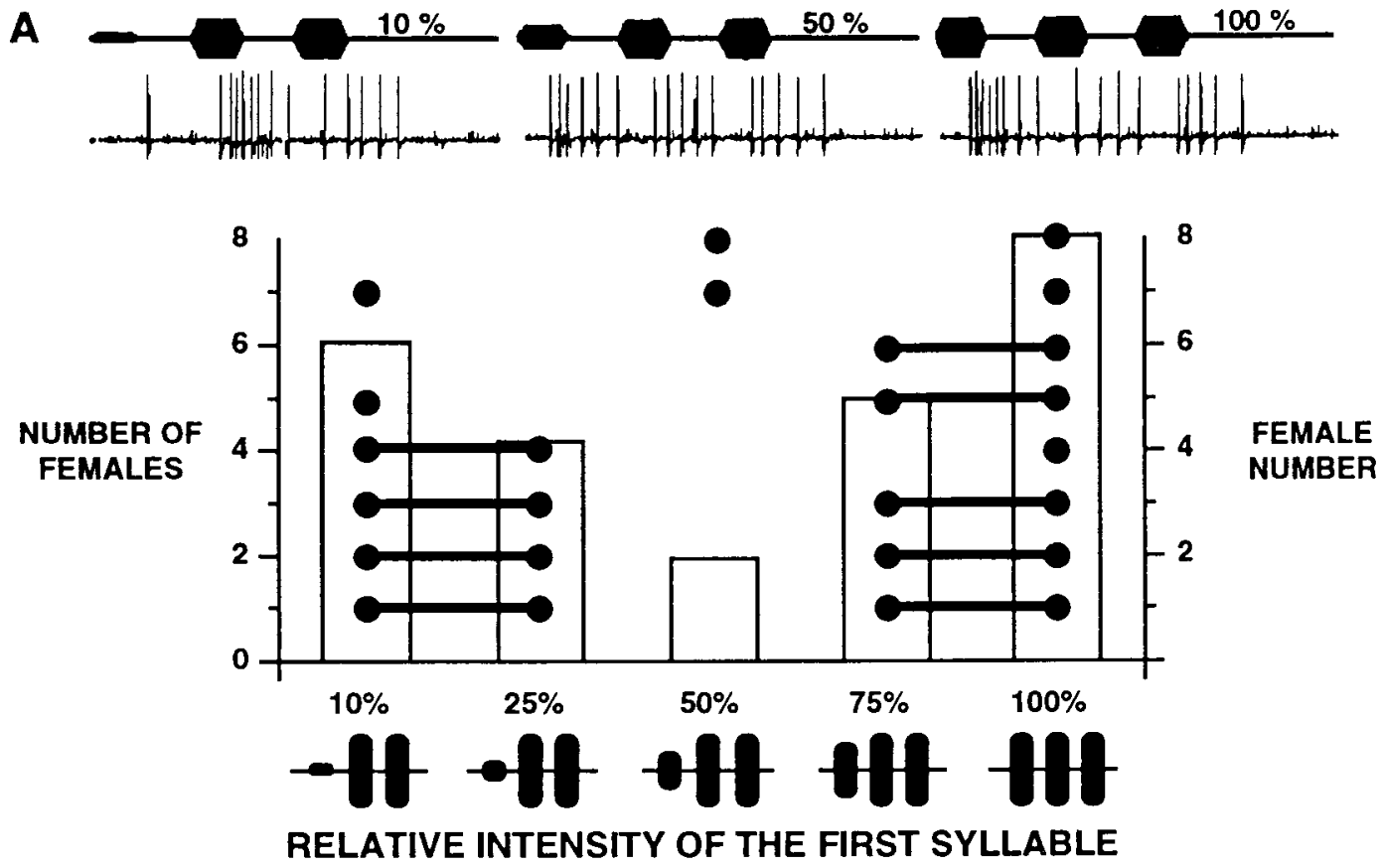

B

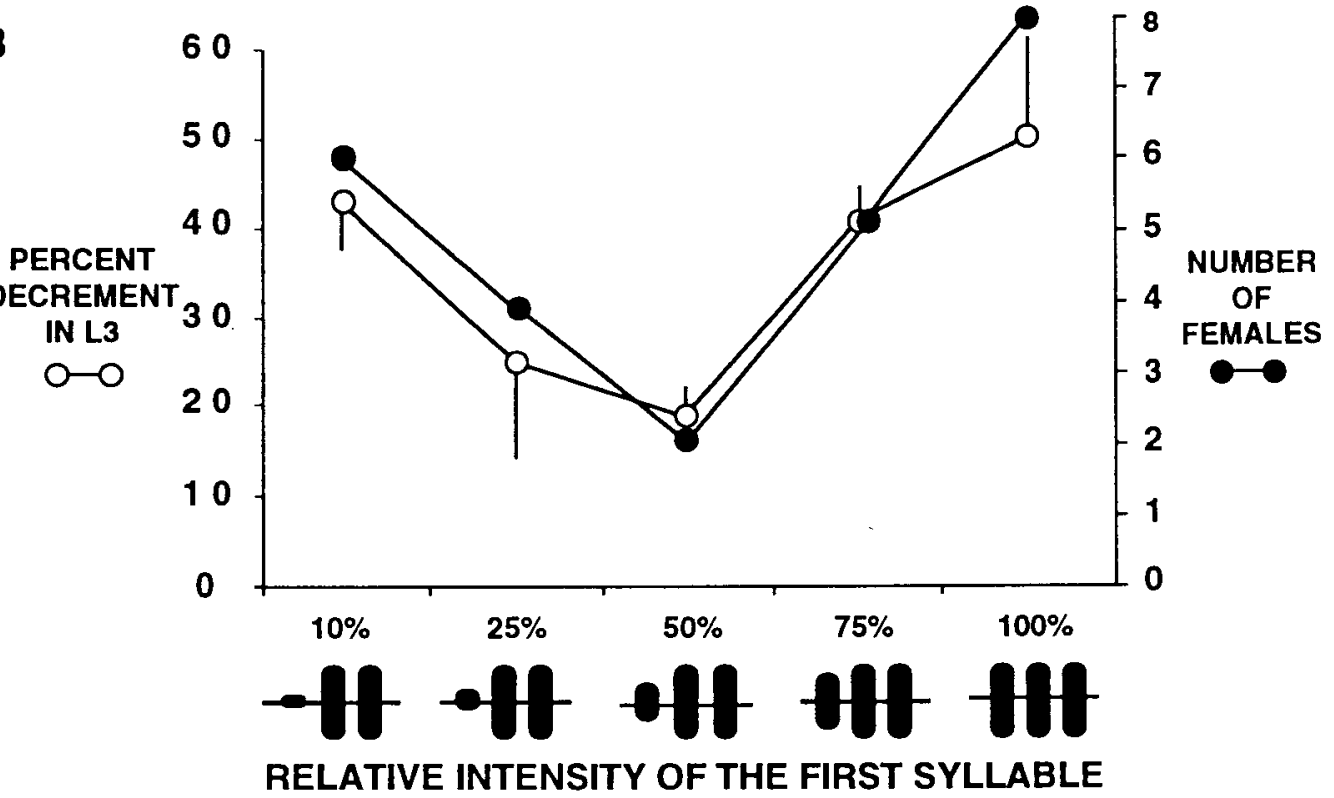

Fig. 2. The correlations between $\mathrm{L} 3$ 's responses to CSs $(50 \mathrm{~ms} \mathrm{SP})$ with amplitude modulated first syllables $(5 \mathrm{kHz}, 90 \mathrm{~dB}$ for $100 \%$ ) and the phonotactic responses of 5-day-old female crickets to the same stimuli. A. Extracellular recordings of an $L 3$ neuron's response to model CSs with the indicated amplitude of the first syllable. The columns show the number of females $(n=8)$ that responded to each model CS. A horizontal row of black dots indicates the responses of a single female to the model CSs. B. A comparison of the mean percent decrement of the $L 3$ neuron's $(n=3)$ responses with the phonotactic response of 8 females to the same stimuli. Bars associated with means indicate the standard errors.

the female's increasing or decreasing phonotactic responsiveness matched very well with L3's increasing or decreasing decrement.

Five-day-old females that were selective for CS SP (data not shown) did not respond phonotactically to CSs with an SP of $90 \mathrm{~ms}$ (Fig. 3A, 100\%). However, all of these females responded to one or more CSs for which the amplitude of the second and third syllables of the model CS was reduced. Response rates were maximal to CSs with second and third syllable amplitudes of $50 \%$ and were smaller as second and third syllable amplitudes decreased or increased from 50\% (Fig. 3A).

When stimulated with CSs having a $90 \mathrm{~ms}$ SP, the L3 neuron responded with little decrement when sec- 
ond and third syllable amplitudes were $100 \%$ and maximal decrement to second and third syllables with amplitudes of $50 \%$ (Fig. 3A, B). Syllables with amplitudes of $10 \%$ (and some syllables with a $25 \%$ amplitude - thus a question mark is shown in Fig. 3B) were below the $\mathrm{L} 3$ neuron's threshold and the neuron responded as if the model CS contained a single syllable. For CSs with second and third syllable amplitudes of 50,75 and
$100 \%$, the change in behavioral attractiveness of the call matched the change in decrement in the L3 neuron's response to these same CSs very well.

In order to understand better the processes that shape L3's decrementing response to model CSs that reproduce SPs characteristic of calling conspecific males, these neurons, in both young and old females, were penetrated and depolarized by positive current

A
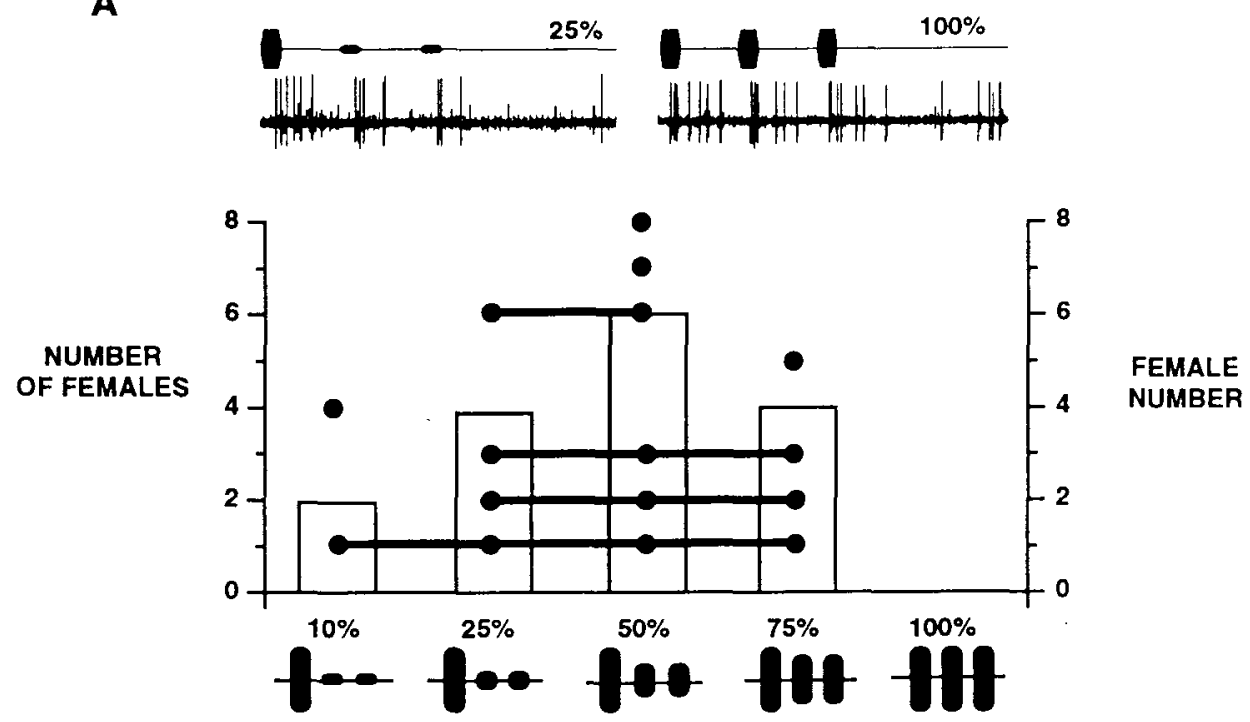

RELATIVE INTENSITY OF THE SECOND AND THIRD SYLLABLE

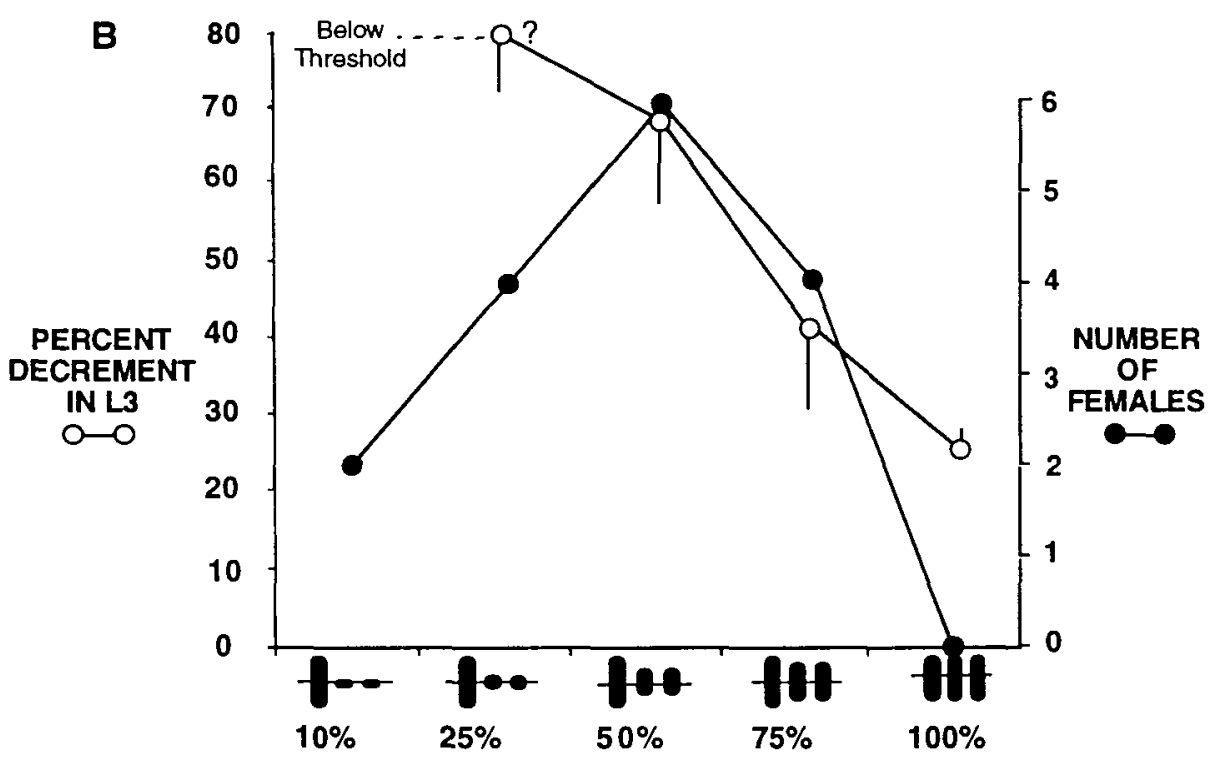

RELATIVE INTENSITY OF THE SECOND AND THIRD SYLLABLE

Fig. 3. The correlations between L3's responses to CSs ( $90 \mathrm{~ms}$ SP) with amplitude modulated second and third syllables (5 $\mathrm{kHz}, 90 \mathrm{~dB}$ for $100 \%$ ) and the phonotactic responses of 5-day-old female crickets to the same stimuli. A. Extracellular recordings of an L3 neuron's response to model CSs with the indicated amplitude of the second and third syllables. The columns show the number of females $(n=8)$ that responded to each model CS. A horizontal row of black dots indicates the responses of a single female to the model CSs. B. A comparison of the mean percent decrement of the L3 neuron's $(n=3)$ responses with the phonotactic response of 8 females to the same stimuli. Bars associated with means indicate the standard errors. 
injection so that they produced action potentials repeatedly. As seen in Fig. 4A, in response to single syllable stimuli presented at both 5 and $16 \mathrm{kHz}$ to young females, there was a clear reduction in spiking in the five overlayed traces of $\mathrm{L} 3$ neurons at the time the response to the next syllable would have occurred (bar marked ' $r$ ' in Fig. 4A). When these same stimuli were presented to old females with nondecrementing L3 neurons, there was no clear pause induced in response to $16 \mathrm{kHz}$ stimuli (or $5 \mathrm{kHz}$ stimuli, data not shown). Thus there is a definite delayed inhibition in the $\mathrm{L} 3$ neurons of young females, that occurs during the time a response to a subsequent syllable (produced at species typical SPs) would occur. This delayed inhibition is not apparent in the response of $\mathrm{L} 3$ neurons in old females that do not respond in a clearly decrementing manner to CSs with species typical SPs.

In general, L3 neurons in old females respond to model CSs with less excitation, particularly in response to the first syllable, than do L3 neurons in young females (Fig. 1D; Henley et al., 1992). In an attempt to evaluate the source for the differences between the responses of $\mathrm{L} 3$ neurons in young and in old females to model CSs, the prothoracic leg (and thus the auditory nerve) contralateral to the recording site in the input dendrites of the L 3 neuron in the opposite hemiganglion was severed. The resulting changes in the responses of $\mathrm{L} 3$ neurons in old females revealed both increased excitation in response to the single syllable CS and the appearance of delayed inhibition that was not apparent during the precut recording from the same neuron (Fig. 4C). These $\mathrm{L} 3$ neurons from old females with severed contralateral auditory nerves responded to 3 syllable CSs ( $50 \mathrm{~ms} \mathrm{SP}$ ) with an increased decrement in spiking (Fig. 4D, decrement increased from $9 \%$ to $40 \%$ after cutting for 5 females) typical of L3 neurons recorded from young females (Fig. 1A, $50 \mathrm{~ms} \mathrm{SP}$ ).

\section{Discussion}

SP-specific responses of the L3 neuron correlate with phonotactic selectiveness for CS SP by female A. domesticus in several ways: a) the L3 neurons of young females respond to model CSs with a SP-selective decrement in spiking that is greatest when stimulated with SPs that are most phonotactically attractive and least to SPs that fall outside of the conspecific male's range of SPs and are behaviorally unattractive (Fig. 1A, Atkins et al., 1989); b) the L3 neurons of old virgin females do not decrement selectively (Henley
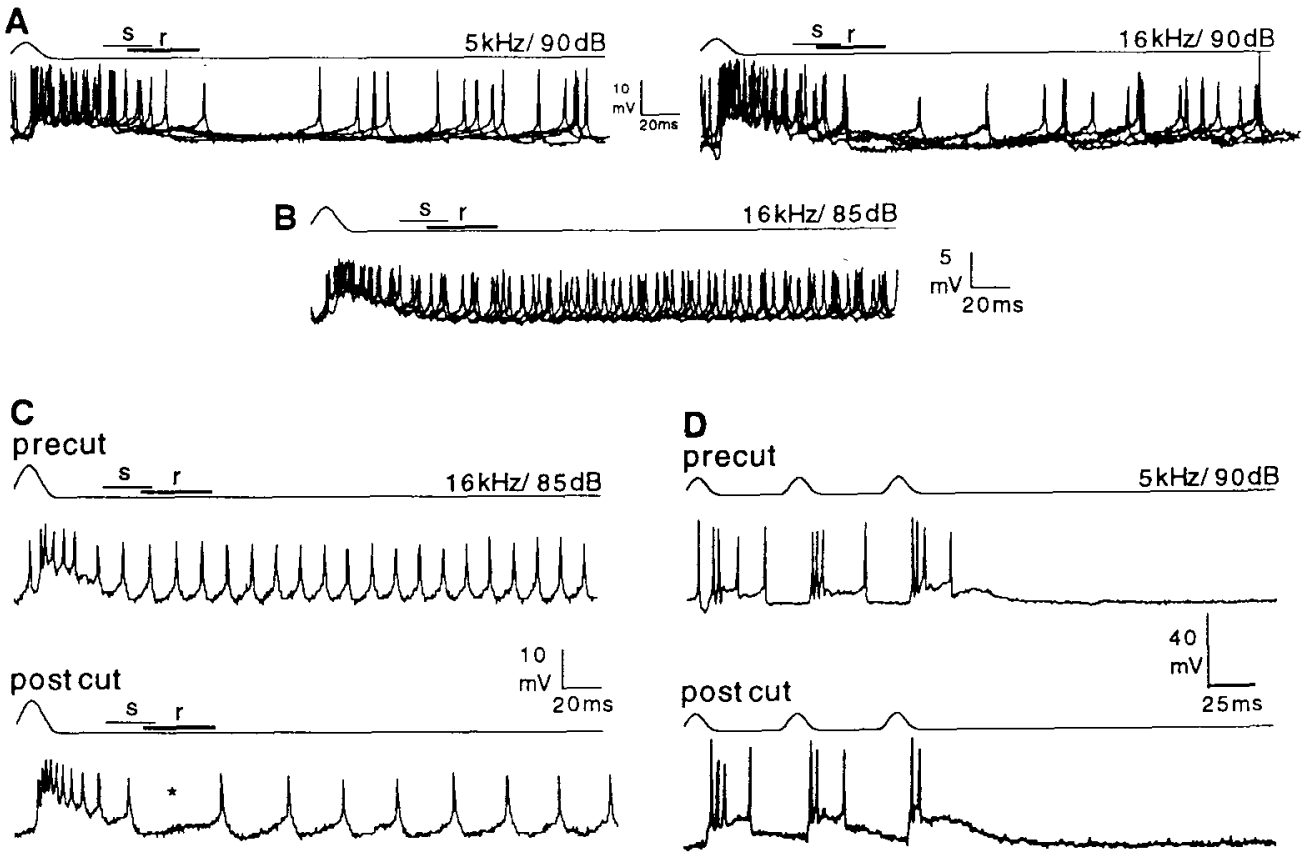

Fig. 4. A. The response of an L3 neuron ( 5 responses, recorded intracellularly from a 5-day-old female, were superimposed) to a single syllable ( $15 \mathrm{~ms}$ duration). The neuron was injected with positive current (.9 $\mathrm{nA}$ ), causing it to fire repeatedly during the interval between stimuli. The bar indicated with an 's' indicates when the next syllable of a $50 \mathrm{~ms}$ SP would occur and the bar indicated with an ' $r$ ' indicates when the response to a second syllable would be expected. B. Recording from a 28-day-old female under the same conditions as part A. C. Recording (under the same conditions as part A) from a 28-day-old female before and after cutting the contralateral prothoracic leg. D. Recording of an L3 neuron from a 28-day-old female responding to a 3 syllable CS (50 $\mathrm{ms} \mathrm{SP)} \mathrm{before} \mathrm{and} \mathrm{after} \mathrm{cutting} \mathrm{the} \mathrm{contralateral} \mathrm{leg.}$ 
et al., 1992) and phonotaxis is unselective for CS SP (Fig. 1B); c) treatment of old females that are behaviorally unselective for CS SP with JHIII restores both their SP selective behavior (Walikonis et al., 1991) and the SP selective responses of the L3 neuron (Henley et al., 1992). These correlations suggest that the L3 neuron may be involved in the recognition of the conspecific male's CS.

Both the $\mathrm{L} 3$ and the $\mathrm{L} 1$ auditory interneurons of female A. domesticus (homologous to the first order AN2 and AN1 auditory interneurons of other cricket species; Hennig 1985, Stout et al., 1985, Stumpner et al., 1995) respond to the male's calling song, encode its temporal structure, and project to similar areas in the brain. However the L1 neuron does not respond selectively to the SPs of model CSs (Stout et al., 1988, Stumpner et al., 1995). Thresholds for the L3 neuron's response to $5 \mathrm{kHz}$ CSs range between 65 and $70 \mathrm{~dB}$ in both young and old females (Atkins et al., 1989, Henley et al., 1992) while L1 thresholds range between 45 and $50 \mathrm{~dB}$ (Stout et al., 1989, Stumpner et al., 1995). Phonotactic thresholds of females match L1 thresholds rather closely in both young (older than 2 . 3 days) and old females (Stout et al., 1991, Walikonis et al., 1991). SP-selective phonotaxis by young female A. domesticus has been consistently evaluated at intensities above L3's threshold (e.g. Walikonis et al., 1991). Preliminary experiments (unpublished) indicate that although young females are phonotactically responsive, they are rather unselective for the SPs of CSs produced at $60.65 \mathrm{~dB}$ (just below L3's thresholds) while these same females demonstrate species typical selectiveness for the SPs of CSs produced at $85 \mathrm{~dB}$. Thus, the SP-selective responses of the L3 neuron may play a very important role in the female's SP-selective phonotaxis. However, clear understanding of the effect of CS intensity on SP-selectiveness is dependent on careful experimental evaluation planned for the near future. Doolan and Pollack (1985) also demonstrated that female Teleogryllus oceanicus are quite unselective for the syllable period of models of the conspecific male's calling that are produced at low $(50.60 \mathrm{~dB})$ intensities than for calls produced at higher intensities.

The L3 neuron responds to all model CSs, not just those that reproduce the SPs characteristic of the conspecific male's CS (Fig. 1A, Henley et al., 1992). Therefore the possible role(s) that L3's SP-selective decrement (Fig. 1A) might play in the young female's phonotactic response to attractive calls was evaluated by a series of experiments in which the amplitude of individual syllables of a model CS were modulated, possibly changing L3's SP-selective decrement and thus the behavioral attractiveness of such a call.

Varying the amplitude of the first syllable of model
CSs with a $50 \mathrm{~ms}$ SP resulted in CSs that were most behaviorally attractive when they maximized the $\mathrm{L} 3$ neuron's decrement (amplitude $=100 \%$, decrement $=$ $50 \%$ ) and least attractive when the L3 neuron responded with minimal decrement (amplitude $=50 \%$, decrement $=20 \%$, Fig. 2A, B). Conversely, a CS with a $90 \mathrm{~ms} \mathrm{SP}$ (outside the range produced by conspecific males) that was behaviorally unattractive to young females and produced minimal decrement in L3's response (amplitude $=100 \%$, decrement $=20 \%$, Fig. $3 \mathrm{~A}$ and $\mathrm{B}$ ) was much more attractive when the amplitudes of the second and third syllables were reduced to a level $(50 \%)$ which resulted in maximal decrement (70\%) by the L3 neuron. Both of these changes in CS attractiveness strongly suggest that the L3 neuron's decrementing response to SP is an important step in the stimulus filtering that leads to recognition of CSs as attractive stimuli by young females. The processing of model CSs that leads to the SP-specific decrementing responses of the $\mathrm{L} 3$ neuron requires a neuronal network in the prothoracic ganglion of young females with temporal information that is tuned to the SPs that are characteristic of the conspecific male's CS.

In response to a single syllable CS, a delayed inhibition occurred at the time (approximately $50 \mathrm{~ms}$ following the onset of the L3 neuron's response to a preceding syllable) that a response to the next syllable of a CS produced with an SP of $50 \mathrm{~ms}$ would occur (Fig. 4A). This delayed inhibition is, at least in part, responsible for L3's SP-selective decrement. Atkins et al. (1989) demonstrated that the SP-selective decrementing response of the $\mathrm{L} 3$ neuron occurred in prothoracic ganglia that were isolated from the rest of the female cricket's central nervous system. Thus, the observed delayed inhibition must result from information stored in the prothoracic ganglion. The effectiveness of the inhibition apparently varies throughout the time interval following its onset and results in the greatest decrement in response to SPs of 50 to $70 \mathrm{~ms}$ (Fig. 1A).

Old virgin females are indiscriminate in their phonotactic responses to CSs with a wide range of SPs and the L3 neuron in old females (Henley et al., 1992) receives less excitation and does not selectively decrement in its responses to CSs with this same range of SPs (Fig. 1B). This loss of selective decrement is apparently due to the absence of clear delayed inhibition (Fig. 4B and C). However, 'cutting through the leg (and thus the auditory nerve) containing the ear contralateral to the ear providing direct input to the L3 neuron immediately increases both the excitatory input and SP-dependent decrement characteristic of L3's responses in young females (Fig. 4C and D). Stimulating only the ear providing direct input to the 
L3 neuron (Stout et al., 1992) has the same effect as cutting away the contralateral ear. Apparently, in old females, a source of inhibition that is dependent on input through the contralateral ear reduces both the excitatory input and the delayed inhibition that are seen in the L3 neuron's response (Figs. $1 \mathrm{~A}$ and $4 \mathrm{~A}$ ) in young females.

The ON1 neuron has been identified as a source of inhibition resulting from stimulation through the contralateral ear in cricket species (e.g. Selverston et al., 1985, Stumpner et al., 1995). In old female A. domesticus inactivation (killed by illumination of a Luciferyellow filled neuron with blue laser, Atkins et al., 1992) of the ON1 immediately enhanced both excitatory input and delayed inhibition while inactivation of the ON1 in young females had little effect on L3's responses (Atkins et al., 1997). Although the ON1 is present and functional in both young and old female A. domesticus (Stumpner et al., 1995), its inhibitory influence on the L3 neuron is greater in old females. These results suggest that the transformation of L3's unselective responses in old female $A$. domesticus to SP-selective responses typical of young females following treatment with juvenile hormone III (Henley et al., 1992) resulted from a hormonally induced reduction in the effectiveness (strength of coupling) of ON1's inhibitory input into L3.

Schildberger (1984) demonstrated that the BNC2a neuron of female $G$. campestris, located in the supraesophageal ganglion, responded as a band pass filter tuned to the conspecific male's CS SPs by producing action potentials at the fastest rate in response to SPs that were most typical of calling males and most phonotactically attractive to females. Their model for recognition of SP proposed that the timing information for low-and high-pass filter neurons, that together shaped BNC2a's response, was resident in the suprasesophageal ganglion. This model does not agree with the behavioral results demonstrated for female A. domesticus (Fig. 3) in which a CS with an initially unattractive SP of $90 \mathrm{~ms}$ became very attractive by modulating the amplitudes of the second and third syllables without changing the model call's SP. Their model also does not predict the transition from behaviorally very attractive to rather unattractive that occurred when the amplitude of the first syllable of a CS with a most attractive SP $(50 \mathrm{~ms})$ was reduced (Fig. 2). Both of these changes in CS attractiveness occurred when syllable amplitudes were used that either maximized (Fig. 3) or minimized (Fig. 2) the decrementing response of the L3 neuron to levels typical of behaviorally attractive or unattractive SPs demonstrated by Atkins et al. (1989) and Henley et al. (1992).
In male gryllids, the CS is organized by a central pattern generator based on temporal information and neuronal circuitry located in the thoracic ganglia (Kutsch and Otto, 1972). Female's may use this information (Hoy et al., 1977) for processing the SP of the conspecific CS. Temporal information in the prothoracic ganglion of female $A$. domesticus is translated into the delayed inhibition leading to the decrementing response of the $\mathrm{L} 3$ neuron to model CSs with attractive, species typical SPs (Fig. 4A). The importance of L3's decrementing response is emphasized by the phonotactic attractiveness of originally unattractive CSs with a $90 \mathrm{~ms}$ SP (outside of the species range, Fig. 3), using syllable amplitudes that also forced L3 to decrement by an amount typical for attractive SPs. This importance is further emphasized by the greatly reduced phonotactic attractiveness of a CS with an initially attractive SP of $50 \mathrm{~ms}$ when the CS was produced with syllable amplitudes that also reduced L3's decrement to a minimum characteristic of unattractive SPs (Figs. 1 and 2).

In summary, these results indicate that the SP. selective, decrementing response of the $\mathrm{L} 3$ neuron is, very likely, an important first step in the processing of the $C S$, leading to recognition. It may be that since L3's decrementing response is selective for CSs with SPs that are behaviorally attractive, further processing is based on the decrement rather than on specific temporal information on SP stored in the brain of the female cricket as proposed by Schildberger (1984).

\section{Acknowledgments}

This research was supported by National Science Foundation grant No. IBN 92-22129.

\section{References}

Atkins, G., Kessler, D. and Stout, J. (1998) The roles of ON1 and L3 neurons in syllable period selective phonotaxis by female crickets Acheta domesticus. Submitted to J. Comp. Physiol.

Atkins, G., Henley, J., Handysides R. and Stout, J. (1992) Evaluation of the behavioral roles of ascending auditory interneurons in calling song phonotaxis by the female cricket (Acheta domesticus). J. Comp. Physiol., 170, 363-372.

Atkins, S., Atkins, G. and Stout, ]. (1989) Influence of syllable period on song encoding properties of an ascending prothoracic interneuron in the cricket Acheta domestica. J. Comp. Physiol, $165,827-836$.

Doherty, J. A. (1985) Trade-off phenomena in calling song recognition and phonotaxis in the cricker, Gryllus bimaculatus (Orthoptera Gryllidae). J. Comp. Physiol, 156, 787-801.

Doolan, J. and Pollack, G. (1985) Phonotactic specificity of the cricket Teleogryllus oceanicus: intensity-dependent selectivity for temporal parameters of the stimulus. J. Comp. Physiol., 157, 223-233.

Henley, J., Greenwood, J. Stout, J. and Atkins, G. (1992) Age-correlated changes and juvenile hormone III regulation of the syllable period specific responses of the L 3 auditory interneurons in the cricket, Acheta domesticus. J. Comp. Physiol., 170, 373. 378 . 
Hennig, R. (1988) Ascending auditory interneurons in the cricket Teleogryllus commodus (Walker): Comparative physiology and direct connections with afferents. J. Comp. Physiol, 163, 135. 143.

Hoy, R., Hahn, J. and Paul, R. (1977) Hybrid cricket auditory behavior: evidence for genetic coupling in animal communication. Science, 195,82-84.

Kutsch, W. and Otto, D. (1972) Evidence for spontaneous song production independent of head ganglia in Gryllus campestris L.J. Comp. Physiol., 81, 115-119.

Pires, A. and Hoy, R. (1992) Temperature coupling in cricket acoustic communication. II. Localization of temperature effects on song production and recognition networks in Gryllus firmus. J. Comp. Physiol., 171, 79-92.

Pollack, G. and Hoy, R. (1981) Phonotaxis to individual thythmic components of a complex cricket calling song. J. Comp. Physiol., 144, 367-373.

Schildherger, K. (1984) Temporal selectivity of identified and auditory neurons in the cricket brain. J. Comp. Physiol., 155, 171 . 185.

Selverston, A., Kleindienst, H. and Huber, F. (1985) Synaptic connectivity between cricket auditory interneurons as studied by selective photoinactivation. J. Neurosci., 5, 1283-1292.

Stout, J., DeHaan, C. and McGhee, R. (1983) Attractiveness of the male Acheta domesticus calling song to females. I. Dependence on each of the calling song features. J. Comp. Physiol, 153, 509-521.

Stout, J., Atkins, G. and Burghardt, F. (1985) The characterization and possible importance for phonotaxis of ' $L$ '-shaped ascending acoustic interneurons in the cricket (Acheta domesticus). In: Acoustic and Vibrational Communication in Insects, ed. Kalmring and Elsner, pp 89-100. Hamburg: P. Parey.

Stout, J., DeHaan C., Hall, J. and Rhodes M. (1988) Processing of calling songs by an $\mathrm{L}$-shaped neuron in the prothoracic ganglion of the female cricket, Acheta domesticus. Physiol. Entomol., 13, 89-101.

Stout, J. and McGhee, R. (1988) Attractiveness of the male Acheta domesticus calling song to females. II. The relative importance of syllable period, intensity and chitp rate. J. Comp. Physiol., $164,277.287$.

Stout, J., Zacharias, D. and Atkins, G. (1991) Regulation of cricket phonotaxis through hormonal control of the threshold of an identified auditory neuron. J. Comp. Physiol., 169, 765-77.

Stout, J., Hayes, V., Zacharias, D., Henley, J., Stumpner, A. and Atkins, G. (1992) Juvenile hormone controls phonotactic responsiveness of female crickets by genetic regulation of the response properties of identified auditory interneurons. Insect juvenile hormonal research: fundamental and applied approaches, ed. Mauchamp, Couillaud and Baehr, pp 265-283. Paris: INRA.

Stumpner, A., Atkins, G. and Stout, J. (1995) Processing of unilateral and bilateral auditory inputs by the ON1 and L1 interneurons of the cricket Acheta domesticus in comparison to interneurons of other cricket species. J. Comp. Physiol., 178, 15-23.

Thorson, J., Weber, T. and Huber, F. (1982) Auditory behavior of the cricket. 11. Simplicity of calling song recognition in Gryllus, and anomalous phonotaxis at abnormal carrier frequencies. J. Comp. Physiol., 146, 361-378.

Walikonis, R., Schoun, D., Zacharias, D., Henley, J., Coburn, P. and Stout, J. (1991) Attractiveness of the male Acheta domestica calling song to females. III. The relation of age-correlated changes in syllable period recognition and phonotactic threshold to juvenile hormone 111 biosynthesis. J. Comp. Physiol., 169, 751-764. 